\title{
Volvulus, gangrene and perforation of transverse colon in an antepartum eclamptic pregnant patient post caesarean: a case report
}

\author{
Jharna Behura $^{1}$, Sanjiva Kumar ${ }^{2 *}$, Poonam Bagga ${ }^{1}$, Maruti Sinha ${ }^{1}$
}

\begin{abstract}
${ }^{1}$ Department of Obstetrics and Gynecology, Kasturba Hospital, Delhi, India
${ }^{2}$ Department of Surgery, Kasturba Hospital, Delhi, India
\end{abstract}

Received: 16 June 2020

Accepted: 08 July 2020

\section{*Correspondence:}

Dr. Sanjiva Kumar,

E-mail: sanjivakr@gmail.com

Copyright: ( $\odot$ the author(s), publisher and licensee Medip Academy. This is an open-access article distributed under the terms of the Creative Commons Attribution Non-Commercial License, which permits unrestricted non-commercial use, distribution, and reproduction in any medium, provided the original work is properly cited.

\begin{abstract}
Transverse colon volvulus leading to gangrene and perforation is an extremely uncommon acute surgical complication in pregnancy and puerperium, with a very high rate of mortality and morbidity. Surgical resection is the only treatment. Authors present a case of a 20 years. old women, with antepartum eclampsia, who underwent caesarean section for fetal distress. She complained of abdominal pain from second post-operative day. The pain increased in severity and on the third postoperative day, she developed sudden abdominal distention and appeared pale. Ultrasound revealed free intraperitoneal fluid suggestive of hemoperitoneum. An emergency laparotomy was performed. There was about a litre of blood in the peritoneal cavity with clots. The caesarean incision site, uterus adnexa and broad ligaments were all intact. On further exploration, volvulus of the transverse colon was seen along with gangrene and perforation of the middle part of transverse colon. Resection of the gangrenous part was done with closure of the distal loop. Proximal loop was brought out as colostomy. The patient had an uneventful postoperative course thereafter and was discharged on regular diet on $12^{\text {th }}$ postoperative day. Volvulus of transverse colon leading to gangrene is rare in pregnancy and puerperium, but must form part of clinician's differential diagnosis when encountering a patient with persistent abdominal pain and bowel distention. Early diagnosis and timely surgical intervention could significantly improve the outcome of this catastrophic condition.
\end{abstract}

Keywords: Outcome, Pregnancy, Transverse colon volvulus

\section{INTRODUCTION}

The reported incidence of intestinal obstruction complicating pregnancy varies from 1 in 1500 to 1 in 66431 births. ${ }^{1,2}$ Large bowel volvulus accounts for 10$30 \%$ of all large bowel obstruction and occurs most commonly in sigmoid colon. Volvulus of transverse colon is quite rare, accounting for $4-11 \%$ of all reported cases. Transverse colon volvulus was first reported by Kallio. ${ }^{3}$ It is associated with a greater mortality than the more common sigmoid or caecal volvulae. Volvulus occurring in pregnancy and puerperium poses a complex problem of delay in presentation and diagnosis, leading to ischaemic necrosis and perforation requiring prompt surgical intervention, bowel resection and colostomy as seen in most of the reported cases. ${ }^{4-6}$ Diagnosis is often delayed due to delay in presentation, poor anticipation of the rare condition, a cautious use of radiological investigation in pregnancy and attributing the clinical feature to other ailments of pregnancy. Perforation, peritonitis and sepsis are the chief complications that cause maternal and fetal morbidity and mortality if intervention is not done early.

Authors present a complicated case of transverse colon volvulus with gangrene and perforation in a patient with antepartum eclampsia, post caesarean, requiring emergency laparotomy and bowel resection. 


\section{CASE REPORT}

A 20-year-old primigravida, presented with labour pains at 39 weeks of gestation to the hospital labour ward. She was a booked case and had no significant past medical or surgical history. On admission her Pulse rate was 96/min, BP - 130/90, no pallor, icterus cyanosis or pedal oedema was seen. Her chest was clear and heart sounds were normal. On abdomen examination she had a term, single live fetus, in cephalic presentation with a fetal heart rate was $146 / \mathrm{m}$, with mild contractions. On pelvic examination, she was found to be in early labour. Her Laboratory workup findings were; hemogram - $12.2 \mathrm{gms}$, platelet count 2.78 lakhs/ml, urine analysis (protein 1+). Biochemical laboratory tests: serum aspartate amino transaminase (AST), $51 \mathrm{IU} / \mathrm{L}$; serum alanine amino transaminase (ALT), 89 IU/L; serum alkaline phosphatase $514 \mathrm{IU} / \mathrm{L}$; serum urea $18 \mathrm{mg} / \mathrm{dl}$ and Serum creatinine $0.7 \mathrm{mg} / \mathrm{dl}$. Her coagulation profile was normal. Few hours later she had a generalized tonic clonic seizure for which Magnesium sulphate regimen was started. Her labour was augmented with artificial rupture of membrane (ARM) followed by syntocinon. She progressed well but at $6 \mathrm{~cm}$ dilatation there was fetal tachycardia with persistent late decelerations for which LSCS was done. A female child of $2.8 \mathrm{~kg}$ was delivered with a good Apgar score; liquor had grade 2 meconium. Patient had another episode of tonic clonic seizure intraoperatively and was managed.

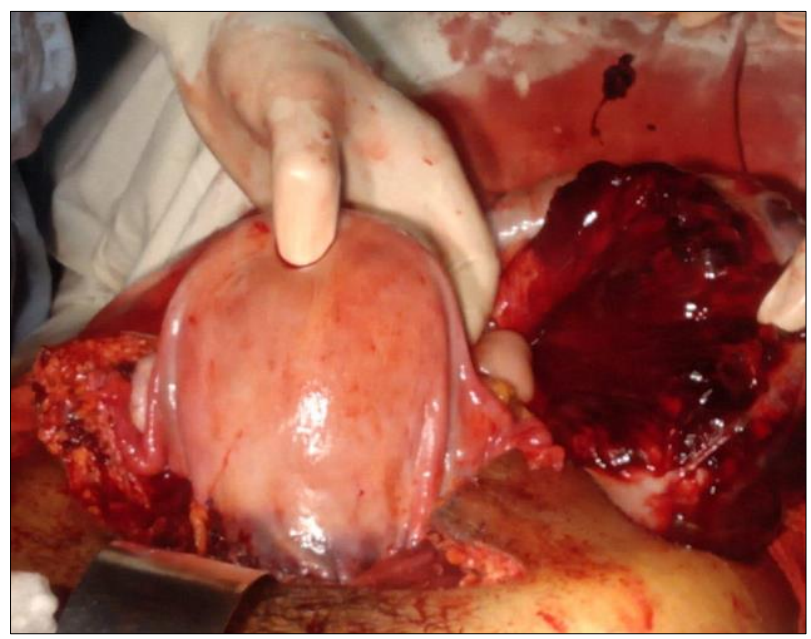

Figure 1: Stitch line of uterine incision intact. Both sides adnexa found normal along with volvulus of large bowel.

Patient was doing well postoperatively and perforation. However, on second post-operative day patient complained of abdominal pain gradually increasing in intensity for which she was kept nil orally and on IV fluids, antibiotics and analgesics. The pain did not subside. On third postoperative day, there was severe pain, distention of abdomen with absent bowel sounds and patient appeared pale. An urgent ultrasound done revealed moderate amount of free fluid in the abdomen.
Presuming hemoperitoneum, patient was taken up for emergency exploratory laparotomy. Blood mixed clots of about a liter was present in the peritoneal cavity. Uterine incision was intact. Bladder, adnexa and broad ligament were intact (Figure 1). On exploring futher, with surgical assistance, it was seen that there was a volvulus of the transverse colon along with a gangrene and perforation of the middle part (Figure 2, 3). Gangrenous part was resected and sent for histopathology examination. Distal loop of colon was closed. Proximal loop was brought out as colostomy. Peritoneal lavage was done. Drain was placed in pelvis; colostomy bag was attached. Abdomen was closed in layers. The patient received 2 units of blood transfusion intraoperatively and 2 units in postop and was shifted to ICU. She recovered well and was discharged on $12^{\text {th }}$ day with normal diet and advised to report after 6 weeks for ileostomy closure.

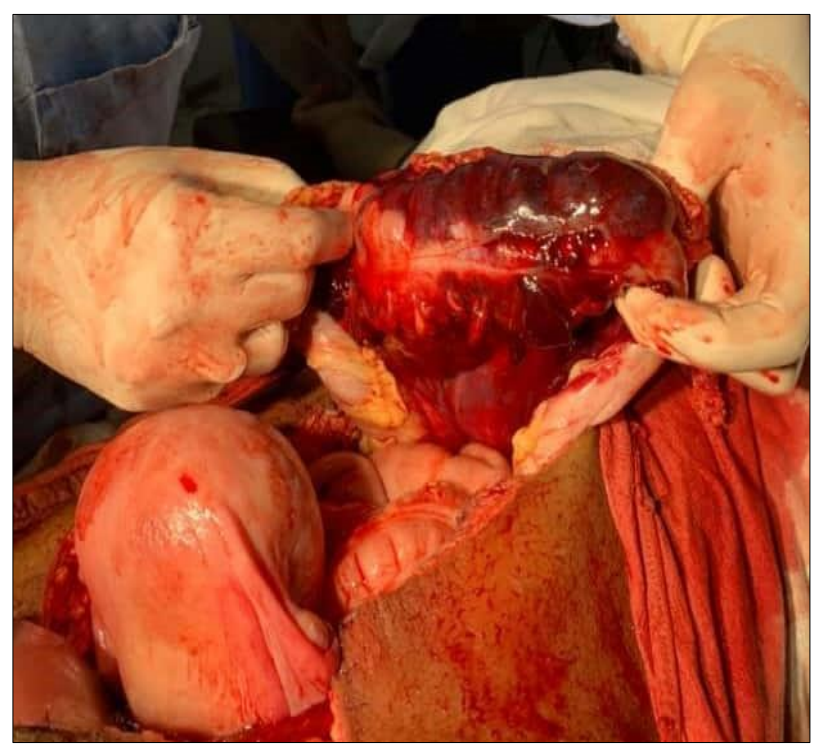

Figure 2: Ischemic necrosis and gangrene of transverse colon.

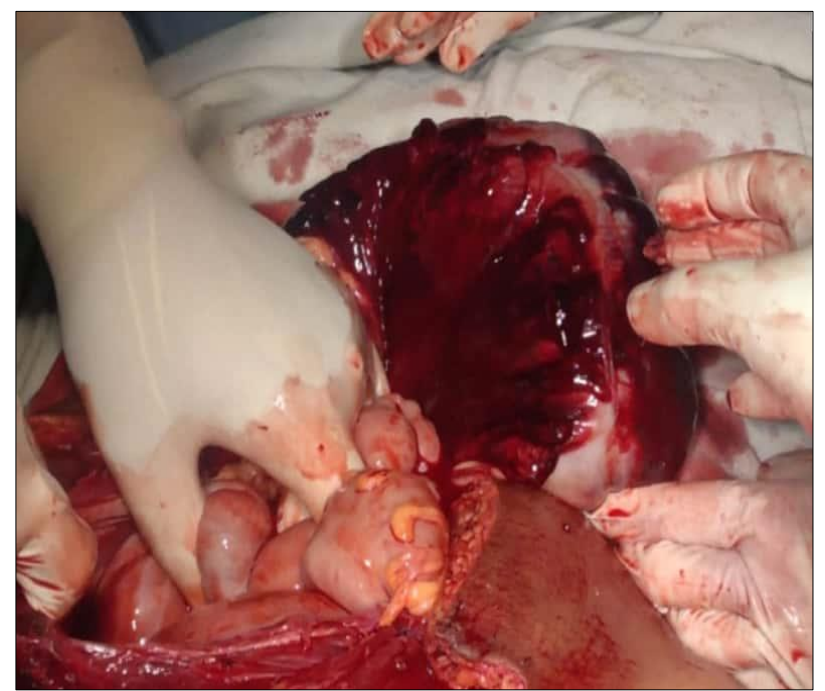

Figure 3: Perforation of the transverse colon. 


\section{DISCUSSION}

Intestinal obstruction in pregnancy and puerperium is a rare event. The major causes are due to adhesions, ventral hernias, Meckel's diverticulum, volvulus, intussusception and cancer colon., 2,78 Even though it is uncommon, intestinal obstruction in pregnancy carries a significant maternal mortality $(6 \%)$ and fetal $(26 \%)$ mortality. ${ }^{9}$ Often it is due to lack of anticipation, as symptoms mimic pregnancy associated problems as happened in this case where the pain abdomen following caesarean did not prompt us to think in terms of a bowel pathology.

Volvulus of intestine is responsible for $25 \%$ of acute bowel obstruction in pregnant women. Sigmoid colon volvulus is the most common cause of bowel obstruction in pregnancy accounting for $44 \%$ of cases. Transverse colon volvulus accounts for only $2-4 \%$ of cases but carries a $33 \%$ mortality rate. ${ }^{10,11}$ It occurs most often in the second and third decades of life. ${ }^{10} \mathrm{~A}$ volvulus is generally caused by twisting of the colon on its vascular pedicle causing venous obstruction followed by arterial compromise leading to ischaemia. ${ }^{12}$ The aetiology could be either congenital or acquired. ${ }^{13,14}$ Congenital causes are usually due to midgut malrotation resulting in abnormal fixation, congenital mega colon, elongation and redundancy of transverse colon and narrowing, absence or malfixation of the mesenteries and their attachments, this probably being the aetiology observed in this case as an acquired cause due to adhesions, inflammatory strictures or carcinoma was ruled out. For diagnosis radiography of abdomen and $\mathrm{CT}$ scans of abdomen are useful tools.

Volvulus of transverse colon has been described in literature to be of two types, subacute-progressive or fulminating. ${ }^{15}$ Fulminating volvulus is a more aggressive form, very rapidly progressing due to closed loop obstruction resulting in vascular compromise which happened in this case. ${ }^{16}$ The subacute form presents with more subtle signs of obstruction. Regarding management, transverse colon volvulus must be surgically corrected..$^{17}$ When necrosis and perforation has occurred, resection with primary anastomosis or resection with colostomy or ileostomy needs to be done.

\section{CONCLUSION}

Transverse colon volvulus complicating pregnancy and puerperium is an uncommon and potentially devastating occurrence and should be recognized as a surgical emergency. Diagnosis requires a high index of suspicion in a patient who presents with complaints of abdominal pain and distention following caesarean section. At least a non-obstetric cause must form a part of clinician's differential diagnosis. Clinical suspicion is vital and a joint management between surgeons and obstetricians is crucial. In this case the suspicion of volvulus was not there, but because of growing pallor and distention and the ultrasound findings of moderate amount of free fluid, emergency exploratory laparotomy was undertaken where the actual cause was diagnosed, and appropriate surgical intervention was done in time.

Funding: No funding sources

Conflict of interest: None declared

Ethical approval: Not required

\section{REFERENCES}

1. Coughlan BM, O'Herlihy C. Acute intestinal obstruction during pregnancy. J R Coll Surg. 1978;23:175-7.

2. Perdue PW, Johnson HW, Stafford PW. Intestinal obstruction complicating pregnancy. Am J Surg. 1992;164:384-8.

3. Kallio K. Uber volvulus coli transversii. Acta Chir Scand. 1932;70:39-58.

4. Iwamoto I, Miwa K, Fujino T, Douchi T. Perforated colon volvulus coiling around the uterus in a pregnant woman with a history of severe constipation. J Obstet Gynaecol Res. 2007;33:731-3.

5. Harer WB, Harer WB. Volvulus complicating pregnancy and puerperium; report of three cases and review of literature. Obstet Gynecol. 1958;12:399406.

6. Sascha Dua R, Rothnie ND, Gray EA. Sigmoid volvulus in the Puerperium. Int $\mathbf{J}$ Gynaecol Obstet. 2007;97:195.

7. Kolusari A, Kurdoglu M, Adali E, Yildizhan R, Sahin HG, Kotan C. Sigmoid volvulus in pregnancy and puerperium: a case series. Cases J. 2009;2(1):13.

8. Damore LJ II, Damore TH, Longo WE, Miller TA. Congenital intestinal malrotation causing gestational intestinal obstruction. A case report. J Repord Med. 1997;42:805-8.

9. Perdue PW, Johnson HW Jr, Stafford PW. Intestinal obstruction complicating pregnancy. Am J Surg. 1992;164:384-8.

10. Zinkin LD, Katz LD, Rosin JD. Volvulus of the transverse colon: report of case and review of the literature. Dis Colon Rectum. 1979;22:492-6.

11. Lau K, Miller B, Schache D, Cohen J. A study of large-bowel volvulus in urban Australia. Can J Surg. 2006;49:203-7.

12. Sparks D, Murtaza Y, Chase D, Thomas D. Ischaemic volvulus of the transverse colon: a case report and review of the literature. Cases J. 2008; $1: 174$

13. Rahbour G, Ayantunde A, Ullah M, Arshad S, Kerwat R. Transverse colon volvulus in a 15 year old boy and the review of the literature. World J Emerg Surg. 2010;5:19.

14. Ciraldo A, Thomas D, Schmidt S. A case report: Transverse colon volvulus associated with Chilaiditis syndrome. Internet J Radiol. 2000;1:1.

15. Eisenstat T, Raneri A, Mason G. Volvulus of the transverse colon. Am J Surg. 1977;134:396-9. 
16. Javors B, Sorkin N, Flint G: Transverse colon volvulus: a case report. Am J Gastroenterol. 1986;81:708-10.

17. Fishman EK, Goldman SM, Patt PG, Berlanstein B, Bohlman ME. Transverse colon volvulus: diagnosis and treatment. South Med J. 1983;76:185-9.
Cite this article as: Behura J, Kumar S, Bagga P, Sinha M. Volvulus, gangrene and perforation of transverse colon in an antepartum eclamptic pregnant patient post caesarean: a case report. Int J Reprod Contracept Obstet Gynecol 2020;9:3505-8. 